\title{
An Adaptive Bandwidth Notch Filter for GNSS Narrowband Interference Mitigation
}

\author{
Tu Thi-Thanh Nguyen ${ }^{1}$, Vinh La The ${ }^{1}$, Tung Hai Ta ${ }^{1}$, Hoang-Lan Thi Nguyen ${ }^{2}$, Beatrice Motella ${ }^{3}$ \\ ${ }^{1}$ NAVIS Centre, Hanoi University of Science and Technology, Vietnam \\ 2 School of Information and Communication Technology, Hanoi University of Science and Technology, \\ Vietnam \\ ${ }^{3}$ Istituto Superiore Mario Boella, Italia
}

Correspondence: Tu Thi-Thanh Nguyen, tu.nguyenthithanh2@hust.edu.vn

Manuscript communication: received 15 December 2014, accepted 15 April 2015

\begin{abstract}
The low level of received signals power makes Global Navigation Satellite Systems (GNSS) receivers vulnerable to many classes of disturbing signals. Among them, narrow band interference (NBI) might cause serious receiver performance degradation. Cancellation of NBI can be implemented by using notch filters (NF), which are controlled by two parameters: the notch frequency, which specifies the band center, and the notch bandwidth, which defines the spectrum area to be removed. The literature on the topic focuses on adapting the notch frequency, without estimating the filter bandwidth. This paper proposes a method able to determine both the notch parameters, optimizing the interference suppression. The performance analysis shows a good improvement by using the adaptive bandwidth notch filter.
\end{abstract}

Keywords- Satellite Navigation, GNSS, Radio Frequency Interference (RFI), Adaptive Bandwidth Notch Filter, Interference Mitigation.

\section{INTRODUCTION}

Though the main applications based on the satellite navigation are related to the transports domains, nowadays the Global Navigation Satellite Systems (GNSSs) play a fundamental role in several fields, belonging to very different areas. The tracking of vehicles fleets (trains, trucks, or vessels), the synchronization of communications and energy distribution networks, the logistics, the mapping of the territory and the systems for the coordination of the rescue teams are examples of important civil applications. Within this scenario, the requirements that the GNSS receiver has to fulfill, both in terms of accuracy and continuity, are becoming more and more stringent.

GNSS systems like GPS and Galileo are based on the Direct Sequence-Spread Spectrum (DS-SS) technique, which intrinsically gives them a high level of robustness [1]. At the same time, it has to be considered that the satellite signals arrive at the receiver antenna with an extremely low level of power (approximately $20 \mathrm{~dB}$ below the noise floor). This makes them vulnerable to different types of disturbance, among which the Radio Frequency Interference (RFI) from external sources is one of the main threats.

All the systems transmitting at carrier frequencies close to the band of interest are potential sources of interference for a GNSS receiver, and even small leakages out of their allocated bandwidth can become a threat for the receiver. Even though events of unintentional RFI are generally unpredictable, several cases have been experienced in the past and reported in literature [2-6].
Moreover, the growing number of wireless communication infrastructures increases the probability that some out of band energy affects the performance of GNSS receivers.

Normally, the RF interference is classified as wideband (WB) or narrow-band (NB), depending on the ratio between the interference and the desired GNSS signal bandwidths [7]. In this sense, an interference might be classified differently, depending on the specific GNSS signal that is considered (e.g., the same interference can be classified as wide for the GPS L1 $\mathrm{C} / \mathrm{A}$ code and narrow for the GPS $\mathrm{P}(\mathrm{Y})$ code). The limit for a narrow-band interference is a single tone [7], usually referred to as continuous wave (CW).

In the literature, several examples of proposed solutions able to detect (and eventually mitigate) interfering sources can be found [5, 8-10]. Normally, the monitoring techniques are properly designed to cope with a specific class of disturbances (wideband, narrowband, pulsed signals, etc.). In this paper we focus on the class of narrow-band interferers (NBI), where the term "narrow" has to be considered with respect to the GPS L1 C/A code and the Galileo E1 signals (i.e., the interference bandwidth is within tens of kilohertz).

Considering such a classification and in accordance with [11], for this kind of disturbing signals, the notch filter (NF) has proved to be an efficient mitigation technique, since it can be considered a good compromise between efficiency and complexity. When using a NF to remove narrowband interference, it has to consider its two parameters: notch frequency and notch bandwidth. The notch frequency decides the center frequency to 


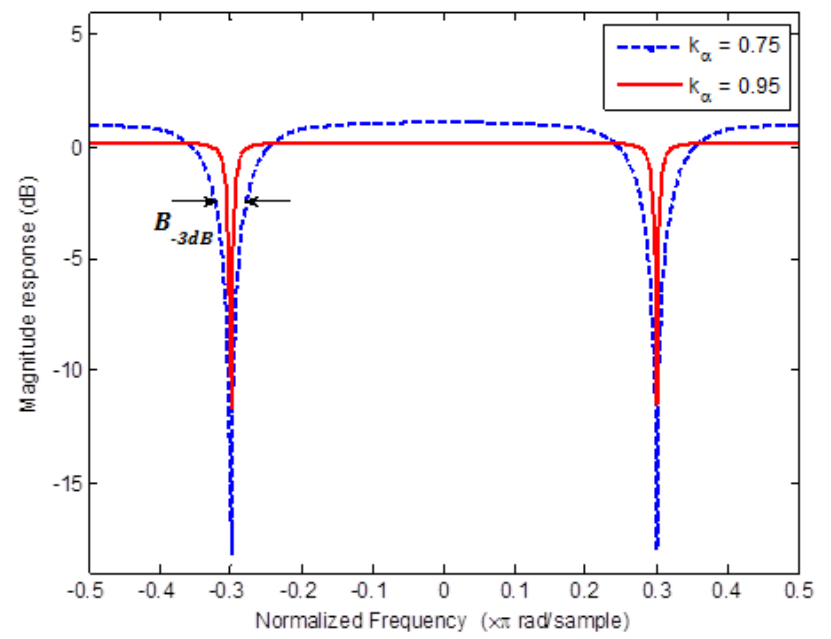

Figure 1. Frequency response of notch filter for two difference pole factors.

be removed. The notch bandwidth is the width of frequency region in which the signal power is reduced by $3 \mathrm{~dB}$. The notch frequency can be adapted and track the interference frequency by using the Least mean square (LMS) algorithm [11]. However, the problem of determining the notch bandwidth has not been considered. To further improve the interference mitigation capability, in this paper, we propose an interference mitigation module based on adaptive notch filtering, which takes into account the adaptation of both the notch frequency and the notch bandwidth. The performance analysis proves an improvement of 1 to $3 \mathrm{~dB}$ for the proposed method with respect to the conventional one in the literature.

As for the paper structure, after this introduction, Section 2 introduces the application of the notch filter in the narrowband interference mitigation. Section 3 proposes two added methods in the interference mitigation module of a GNSS receiver: one for interference bandwidth estimation and the other for the NF configuration. Section 4 gives performance analysis of the proposed module, and Section 5 concludes the paper.

\section{Notch Filter for Narrowband INTERFERENCE MITIGATION}

\subsection{Overview of Notch Filter}

As defined in [12], a notch filter (NF) is a filter that passes all frequencies except those in a stop or rejection band centered on a central frequency. Therefore, a notch filter can be defined by two parameters: the notch bandwidth $B_{N}$, and notch frequency $f_{N}$.

There are several ways to implement a notch filter, normally divided in three main classes: the Fast Fourier Transform (FFT)-based, the Finite Impulse Response (FIR), and the Infinite Impulse Response (IIR) notch filters [12]. In recent years, IIR notch filters are considered for GNSS interference mitigation because of their low computational requirements, efficient implementation and low number of parameters to be adapted [11].
The IIR two-pole NF has the following transfer function [11]:

$$
H(z)=\frac{1-2 \operatorname{Re}\left\{z_{0}\right\} z^{-1}+\left|z_{0}\right| z^{-2}}{1-2 k_{\alpha} \operatorname{Re}\left\{z_{0}\right\} z^{-1}+\left|z_{0}\right| k_{\alpha}^{2} z^{-2}}
$$

where $z_{0}=r e^{j 2 \pi f_{N}}$ is the zero, which corresponds to the notch frequency $f_{N}$, and $k_{\alpha}$ (with $0<k_{\alpha}<1$ ) is the pole contraction factor, which decides the notch bandwidth. To effectively exploit the capability of NF for removing interference signals, the zeros should be constrained to be on the unit circle [13].

Figure 1 reports the frequency responses of a notch filter with two different pole factors. It is clear that the pole factor $k_{\alpha}$ decides the width and the depth of the notch: the more the value of $k_{\alpha}$ is close to unity, the narrower and shallower the notch is. In other words, the notch bandwidth is characterized by the pole factor $k_{\alpha}$, as it decides which frequency components and how much their power will be removed. Hence, the problem of determining $k_{\alpha}$ is very important.

\subsection{GNSS Receiver Structure with NF Interference Mitigation Module}

As mentioned in Section 1, the notch filter has been proved to be an efficient interference mitigation technique. Figure 2(a) shows a high-level block diagram of the GNSS receiver chain, where the Interference detection/estimation block and the Notch filter block are present. After arriving at the receiver's antenna, the GNSS signal (with the noise and the interference components) is filtered, down-converted to the Intermediate Frequency (IF), and sampled by the front-end. Then, the 'Interference Detection and Estimation unit' is in charge of (see Figure 2(b)):

- Deciding if the interference is present (and the notch filter needs to be activated);

- The interference frequency $f_{\text {int }}$ and interference bandwidth $B_{\text {int }}$ are estimated as $f_{\text {int }}$ and $B_{\text {int }}$. Then, the NF parameters $f_{N}$ and $k_{\alpha}$ are calculated based on the estimated values.

If no interference is detected, then the regular signal processing is implemented; otherwise $f_{\text {int }}$ and $B_{\text {int }}$ provided by the "Interference Detection and Estimation Unit" block are used to configure the NF, which is responsible for suppressing the interference components in the IF signal samples.

In reality, the characteristics of the interference signal vary with time. As an example, Figure 3 (from [4]) shows some plots of spectra evaluated (every 18 seconds over a 1 minute of data set) from live signals, collected in a hostile environment. It is evident how the signal spectrum changes significantly over time and several spurious peaks appear always over the same frequency ranges (i.e. [1-3] $\mathrm{MHz}$ and [6-8] $\mathrm{MHz}$ ), but with different widths of band. For this reason, the notch filters are often designed in order to adapt and track the interference signal. The adaptation of the NF towards the interference signal variation along time is performed following the two steps below:

- Step 1: Continuously estimate $f_{\text {int }}$ and $B_{\text {int }}$. 


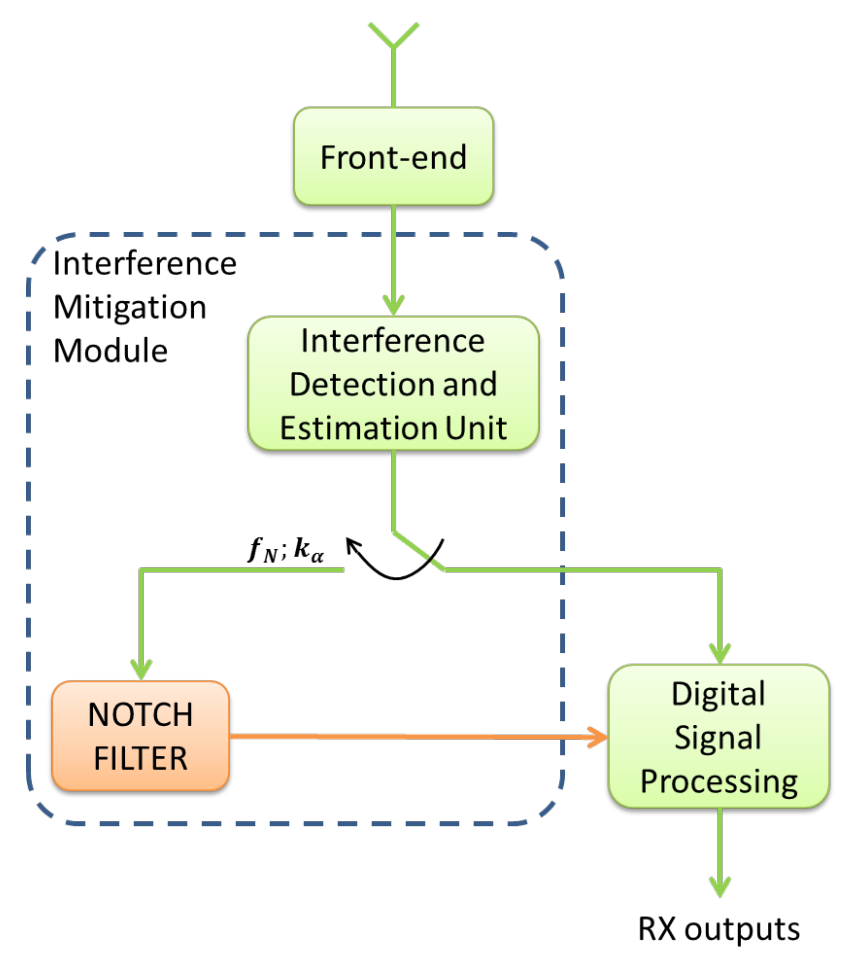

(a) High-level block diagram of the GNSS receiver chain

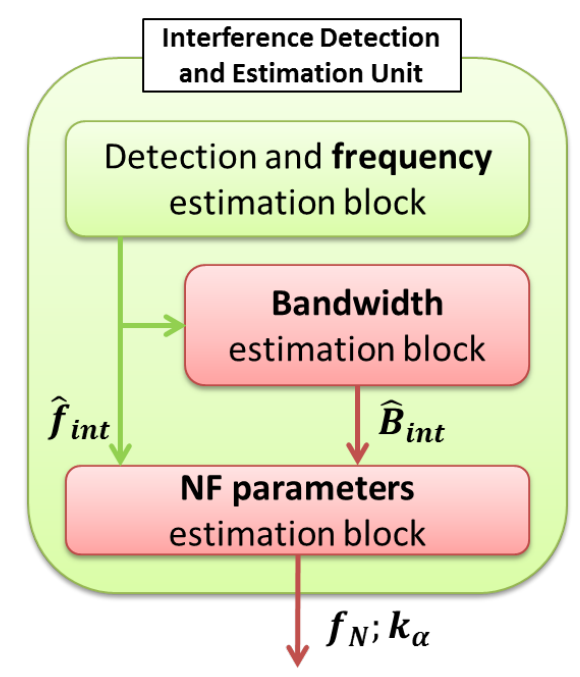

(b) Detection/Estimation Unit

Figure 2. High-level block diagram of the GNSS receiver chain.

- Step 2: Reconfigure the NF via resetting $f_{N}$ and $k_{\alpha}$ based on the estimated values of $f_{\text {int }}$ and $B_{\text {int }}$.

2.2.1 Interference Frequency Estimation (or Interference Detection):

When using NF to remove interference, it is necessary to know the notch frequency (or interference frequency). However, in reality, this parameter is unknown and might vary over time. Thus, an adaptive notch filter (ANF) is designed to estimate and track it automatically. To do this, it is important to note that if the notch frequency matches the interference carrier $\left(f_{N}=f_{\text {int }}\right)$, the amount of removed power will be maximum, and the power of signal after filtering will be minimum. Due to this statement, the ANF can be implemented by using the Least Mean Square (LMS)
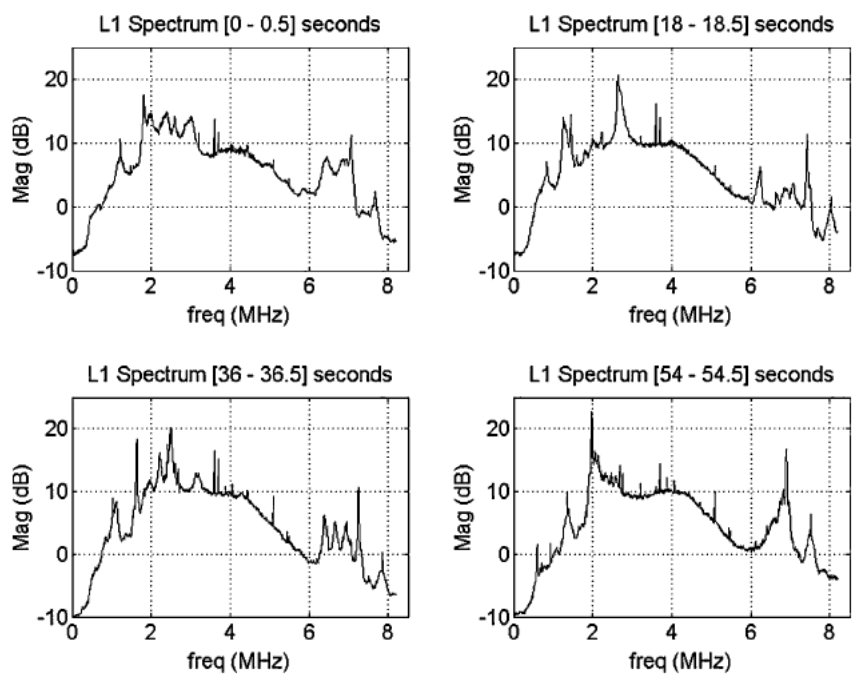

Figure 3. Spectrum at the front-end output at different time instants (from [4]).

algorithm to minimize the power of filter output, as follows [11]. The zero $z_{0}$ of transfer function (1) is updated iteratively. If the interference is present, the angle of $z_{0}$ will converge to the interference frequency. The iterative rule to update $z_{0}$ is

$$
z_{0}[n+1]=z_{0}[n]-\mu \nabla_{z_{0}}\left(|y[n]|^{2}\right),
$$

where $\nabla$ denotes the stochastic gradient, $\mu$ is the algorithm step and $y[n]$ is the output of the notch filter [11]. The estimated interference frequency $f_{\text {int }}$ can be calculated from the mean of $z_{0}$ after convergence.

Since $z_{0}$ is complex, the adaptation is performed with both the real and image part of $z_{0}$. The simulation results showed that if any interference is present in the received signal, the adapted zero converges close to the unit circle. Therefore, [11] introduced a detection unit using the mean value of the amplitude of $z_{0}$ to check if the notch filter is tracking an interference.

This type of filter can be used for both interference detection and mitigation [11]. However, since its zeros are not constrained to unit, this might reduce the capability of the NF for removing interference. Thus, it is only used for the Detection and frequency estimation block in this paper.

\subsubsection{Interference Bandwidth Estimation:}

Besides frequency, bandwidth is also an important characteristic of interference because it relates to the configuration of NF. As explained in Section 2.1, $k_{\alpha}$ decides the width of the removed frequency region. Thus, to determine $k_{\alpha}$, the estimation of the interference bandwidth $B_{\text {int }}$ is necessary. Furthermore, at the same time, not only the interference frequency but also the interference bandwidth can be variable as shown on Figure 3. This illustrates the need for tracking the change of the interference band. However, to the best of our knowledge, there has been no work on the estimation of $B_{\text {int }}$. Therefore, in Section 3 we propose two methods: one for estimating and tracking $B_{\text {int }}$ and the other for estimating $k_{\alpha}$ for the NF from the estimated value. 


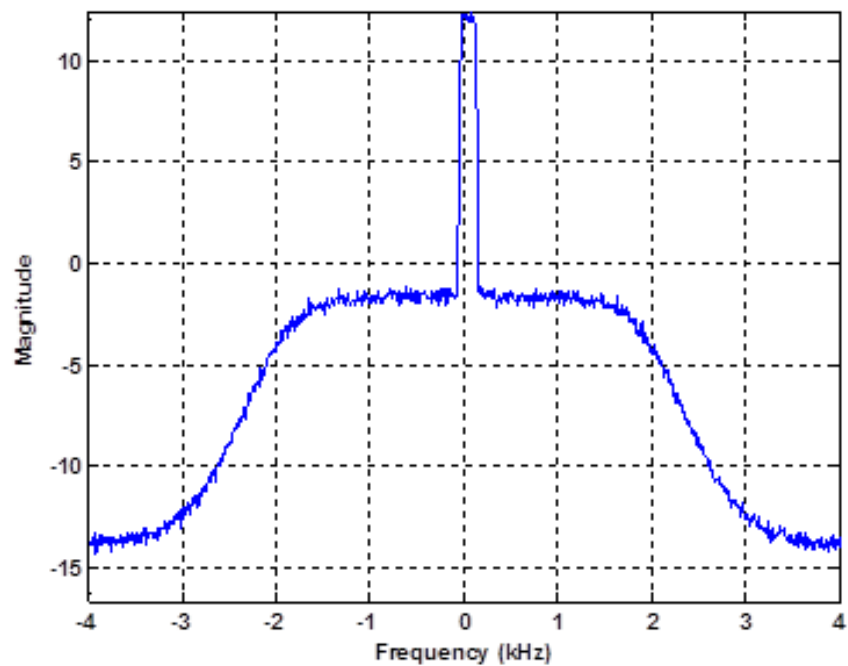

(a) Spectrum of interfered signal

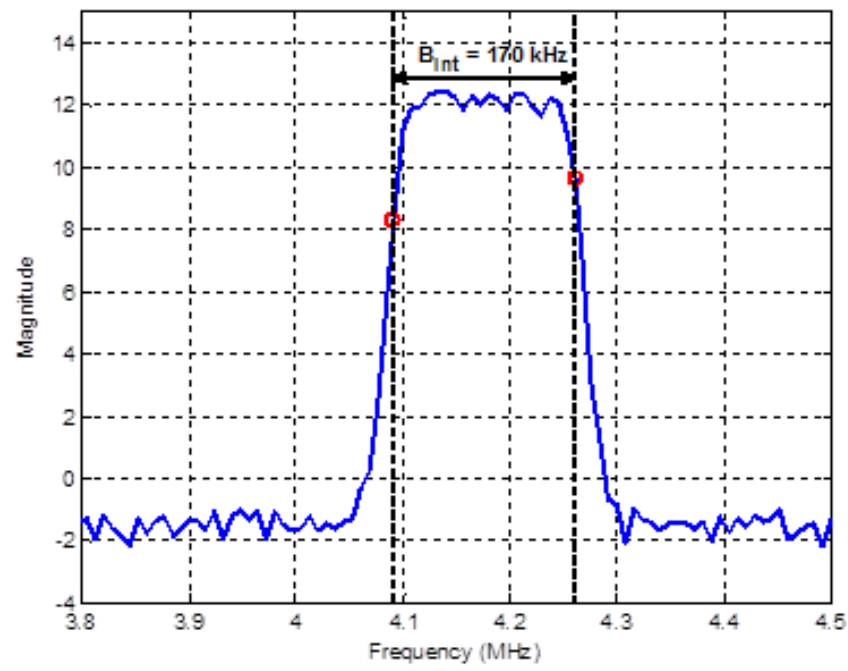

(b) Zoom in the interference band

Figure 4. Spectrum of the input signal.

\section{Proposed Adaptive Bandwidth Notch}

\section{FILTER}

In this section, we introduce the methods to estimate $B_{\text {int }}$ and choose $k_{\alpha}$ for the final NF. They are implemented in the Bandwidth estimation block and NF parameters estimation block in Figure 2(b).

\subsection{Interference Bandwidth Estimation}

Firstly, consider a GPS signal generated by NFUELS [14]. It contains a GPS PRN 1 signal in L1 band, at nominal outdoor signal power: $45 \mathrm{~dB}-\mathrm{Hz}$ of carrier to noise ratio. It is affected by an interference signal with $170 \mathrm{kHz}$ of bandwidth and frequency at $42 \mathrm{kHz}$ from the GPS carrier (the worst interference frequency for PRN 1 [7]). The signal spectrum is shown in Figure 5(a). The $\mathrm{x}$-axis is normalized with respect to the L1 carrier frequency ( $f=0$ corresponds to the GPS band center).

Figure 5(a) shows the signal power at the output of a NF, whose notch bandwidth is fixed at $30 \mathrm{kHz}$ and whose frequency sweeps over the spectrum range.

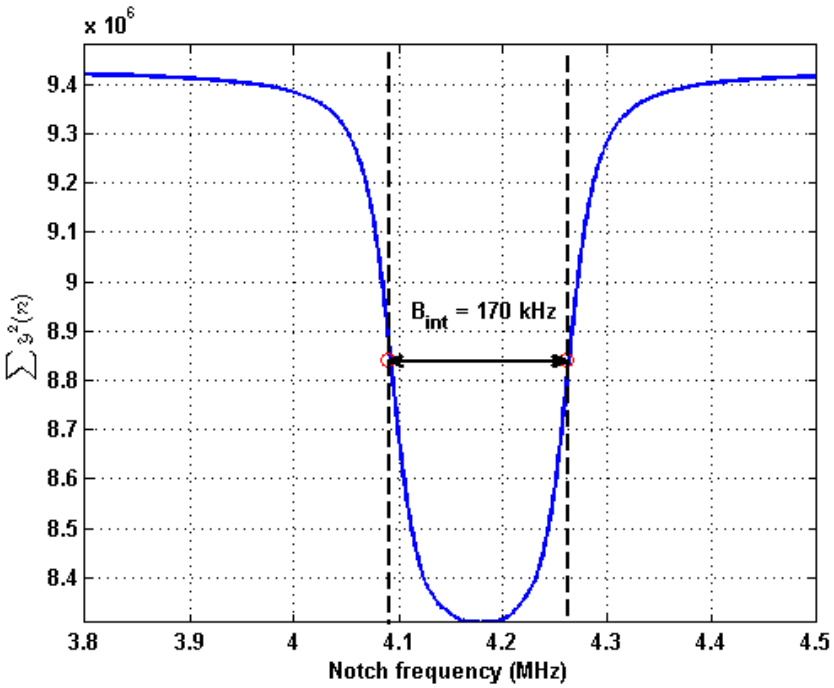

(a) Signal power at the output of a NF

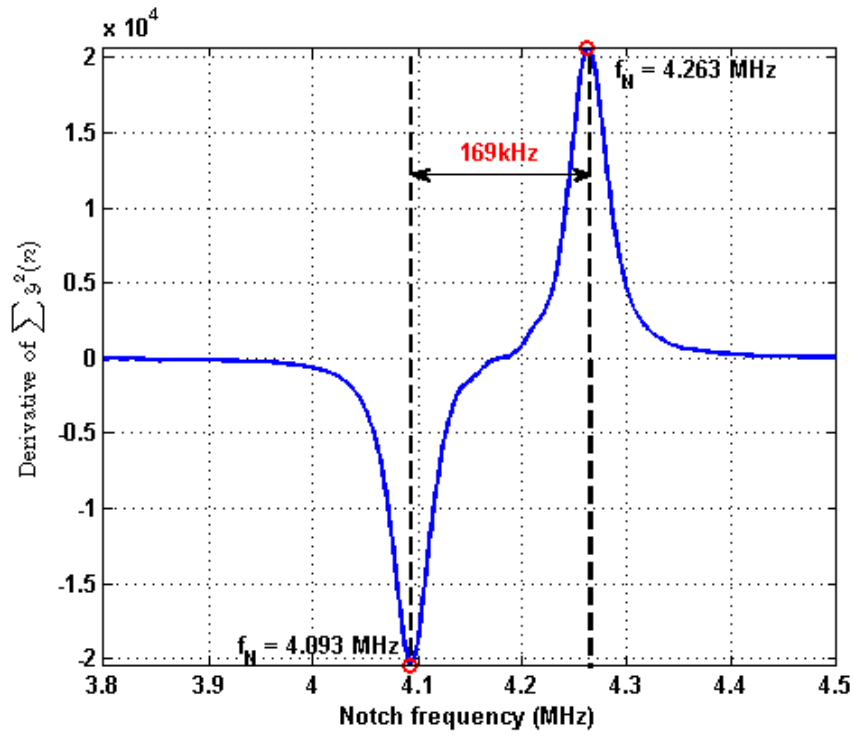

(b) Derivative of the signal power at the output of a NF

Figure 5. The signal power at the output of a NF and its derivative.

The two circles indicate the portion of the spectrum occupied by the interference $\left(B_{\text {int }}=170 \mathrm{kHz}\right)$. Furthermore, Figure 5(b) shows the derivative of the output power (in Figure 5(a)). It is easy to observe how the distance between the two critical points (maximum and minimum) is a good estimation of the interference bandwidth.

On the basis of the described approach, the proposed solution for the bandwidth estimation block follows the next procedure:

- Two notch filters with fixed predefined bandwidth are used with the scope of estimating the interference bandwidth $B_{\text {int }}$. Each NF works on a single portion of the spectrum (i.e., one NF finds the minimum and one NF finds the maximum of derivative of the filter output power), using the interference frequency estimation $f_{\text {int }}$ as the starting point.

- The frequencies at the output of the two notch filters are the limits of the interference band.

This block is presented more clearly in Figure 6 . 


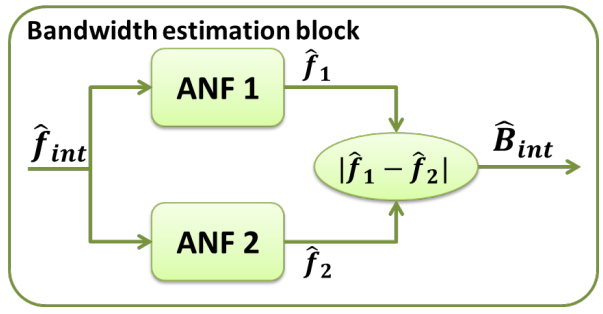

Figure 6. Bandwidth estimation block.

ANF 1 and ANF 2 can be implemented by the same way of the ANF in Section 2.2.1. However, the LMS algorithm is applied to minimize and maximize the derivative of the filter output power. Thus, instead of converging to the interference frequency, they will converge to the two limit frequencies of interference band. To exploit the capacity of interference removal, the zeros of NF in the paper are constrained to unit. Thus, the iterative rule for the updating process in (2) is considered only for the real part of zeros as follows:

$$
\begin{aligned}
\beta[n+1] & =\beta[n] \pm \mu \nabla_{\beta}^{2}\left(y^{2}[n]\right) \\
& =\beta[n] \pm \mu\left[\left(\nabla_{\beta} y[n]\right)^{2}+y[n] * \nabla_{\beta}^{2} y[n]\right],(3)
\end{aligned}
$$

where $\mu$ is the algorithm step [11], and $\beta(1)=$ $\cos \left(2 \pi f_{\text {int }}\right)$. The operation ' - ' is used for the ANF 1 , which performs the maximization, and the operation ' + ' is used for the ANF 2, which performs the minimization.

To reduce the number of variables needed to be adapted, the zeros of transfer function (1) are constrained to be on the unit circle. Thus, $\nabla_{\beta} y[n]$ and $\nabla_{\beta}^{2} y[n]$ can be deduced from $y[n]$ as follows:

$$
\begin{aligned}
y[n]= & 2 \beta[n] k_{\alpha} y[n-1]-k_{\alpha}^{2} y[n-2]+x[n] \\
& -2 \beta[n] x[n-1]+x[n-2] \\
\nabla_{\beta}(y[n])= & 2 k_{\alpha} y[n-1]+2 k_{\alpha} \beta \nabla_{\beta}(y[n-1]) \\
& -k_{\alpha}^{2} \nabla_{\beta}(y[n-2])-2 x[n-1] \\
\nabla_{\beta}^{2}(y[n])= & 4 k_{\alpha} \nabla_{\beta}(y[n-1])+2 k_{\alpha} \beta \nabla_{\beta}^{2}(y[n-1]) \\
& -k_{\alpha}^{2} \nabla_{\beta}^{2}(y[n-2]) .
\end{aligned}
$$

When the LMS algorithm reaches its steady-state, the standard deviation of the output frequency has to be smaller than a threshold T. Moreover, during a convergence period the frequency deviation does not exceed the quarter of the filter bandwidth [15]. Thus, the stop convergence of ANF 1 and ANF 2 can be created when:

$$
\left\{\begin{array}{l}
\sigma\left(\cos ^{-1}(\beta[n])\right) \leqslant T \\
\cos ^{-1}(\beta[D(k)])-\cos ^{-1}(\beta[D(k-1)]) \leqslant \frac{B_{N}}{4}
\end{array}\right.
$$

where $k \in N, B_{N}$ is the bandwidth of filter, $\sigma\left(\cos ^{-1}(\beta[n])\right)$ is the standard deviation of the output frequency during the period of D-iteration. For the simulations in the rest of this paper, $D=5 \times 10^{5}$ and $T=500$ to ensure both the speed and the accuracy of the stop of convergence.
Consequently, the bandwidth of interference can be calculated from the mean values at their output frequency during their convergence period, as follows:

$$
\hat{B}_{\text {int }}=\left|\hat{f}_{1}-\hat{f}_{2}\right|=\frac{\left|\cos ^{-1}\left(\bar{\beta}_{\text {ANF1 } 1}\right)-\cos ^{-1}\left(\bar{\beta}_{\text {ANF1 }}\right)\right|}{2 \pi}
$$

where the bar symbol denotes the mean value.

\subsection{Adaptive Bandwidth Notch Filter Configuration}

As point out in the Appendix, the carrier to noise ratio $C / N_{0}$ of the received signal after filtering can be expressed as

$$
\frac{C}{N_{0}}=\frac{\frac{C_{s}}{N_{0}}\left|\int_{0}^{\frac{F_{s}}{2}} H(f) S(f) d f\right|^{2}}{\int_{0}^{\frac{F_{s}}{2}}|H(f)|^{2} S(f) d f+\frac{C_{i}}{N_{0}} \int_{f_{\text {int }}-\frac{B_{\text {int }}}{2}}^{f_{\text {int }}+\frac{B_{\text {int }}}{2}}|H(f)|^{2} S(f) d f}
$$

where $F_{S}$ is the sampling frequency, $H(f)$ is the transfer function of filter, $S_{i}(f)$ and $S(f)$ are the normalized power spectral density of the interference and the GNSS signal, $C_{s}, C_{i}$ are the power of the received GNSS signal and the interference signal, respectively, $B_{\text {int }}$ is the bandwidth of interference and $f_{\text {int }}$ is the interference frequency.

Figure 7 shows the theoretical line of $C / N_{0}$ with a typical signal available to the commercial GPS receiver with interference bandwidth $B_{i n t}=25 \mathrm{kHz}$ and $P_{i} / P_{s}=$ $30 \mathrm{~dB}$ (data demodulation threshold for the width-band interference) [16]. The interference frequency matches the GPS signal frequency. Since the large $k_{\alpha}$ leads to the distortion of the useful signal, $k_{\alpha}$ in the range $[0.9,1)$ is often chosen in experiments [11, 13, 17]. [17] recommended that $k_{\alpha}$ should be set at 0.9 . In this experiment, the notch filter is investigated with $k_{\alpha}$ larger than 0.9. It can be seen that the best $k_{\alpha}$ is about 0.97 .

According to [17], the relation between $k_{\alpha}$ and the notch bandwidth can be estimated as follows:

$$
B_{N}=\frac{F_{S}\left(1-k_{\alpha}\right)}{\pi} .
$$

Thus, $k_{\alpha}$ of 0.97 corresponds to the notch bandwidth of about $155 \mathrm{kHz}\left(F_{s}=16.3676 \mathrm{MHz}\right)$. This value does not match the interference bandwidth, because $C / N_{0}$ depends on not only the interference bandwidth, but also the power of interference. Thus, it is not possible to use the estimated interference bandwidth to calculate $k_{\alpha}$ directly by (9). In this paper, $k_{\alpha}$ will be estimated based on the expression (8).

It has to note that there are four parameters to be considered in (8): (i) the power of the useful signal $P_{S}$, (ii) the noise density $N_{0}$, (iii) the interference bandwidth $B_{\text {int }}$ and frequency $f_{\text {int }}$, and (iv) the power of interference $P_{i}$. However, $P_{S}$ does not change the shape of the $C / N_{0}$ line, so it is not necessary to know this value in advance. $N_{0}$ can be calculated from the temperature of the environment. $f_{\text {int }}$ and $B_{\text {int }}$ are provided by the Detection and estimation unit. and $P_{i}$ can be estimated by spectrum analysis and refined after signal processing. In this section, these parameters are assumed to be 


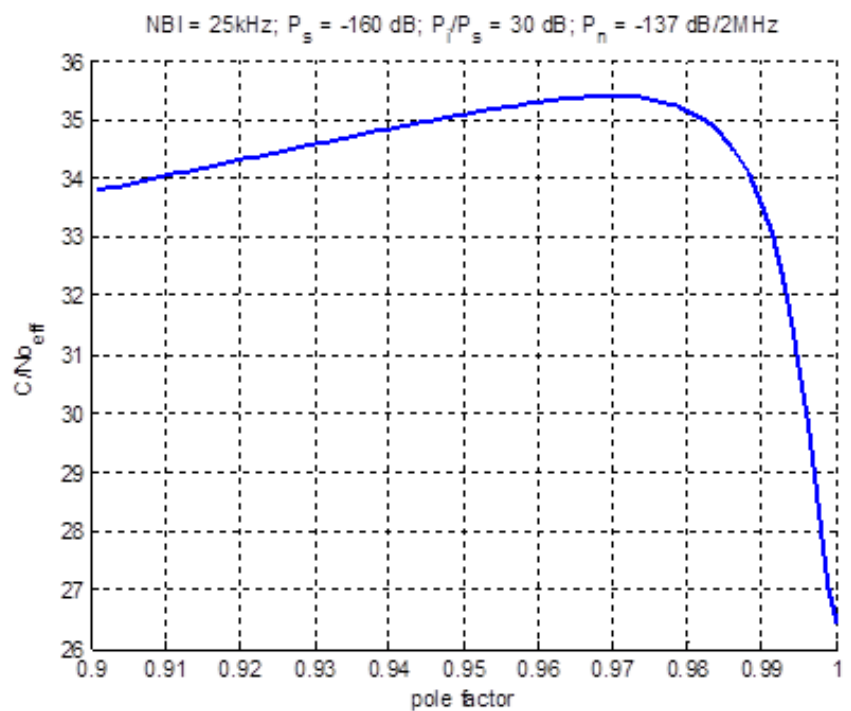

Figure 7. Theoretical $C / N_{0}$ when bandwidth of interference $B_{\text {int }}=$ $25 \mathrm{kHz}$ (ACARS Harmonics) and $P_{i} / P_{s}=30 \mathrm{~dB}$.

known in advance. Now, (8) has only one variable $k_{\alpha}$. However, it is difficult to find the value of $k_{\alpha}$ directly from

$$
\hat{k}_{\alpha}=\arg \max _{k_{\alpha}}\left\{\frac{C}{N_{0}}\left(k_{\alpha}\right)\right\}
$$

Fortunately, since the pseudo-range code of GNSS signal has the line spectrum spaced at $1 \mathrm{kHz}$, Equation (8) can be discretized and calculated fast and easily with varying $k_{\alpha}$. Thus, the problem (10) can be solved approximately by the numerical method. Then, the configuration of NF can be completed.

\section{Performance Analysis}

\subsection{Bandwidth Estimation}

In this section, the simulation results obtained by implementing the bandwidth estimation block will be presented. The characteristics of the input signal are as introduced in Section 3.1. The notch bandwidth of the two ANFs is fixed at $30 \mathrm{kHz}$. The estimated frequencies at their output are shown in Figure 8. The two black lines correspond to the actual limits of the interference bandwidth, while the red and blue lines are their estimation.

Figure 9 shows the achieved result with the simulated signal, which has the configuration as in Section 3.2. Similar to Figure 9, ANF 1 and ANF 2 work well on estimating the interference bandwidth.

The stop of convergence is also shown in Figures 8 and 9. In the case of a wide narrow-band interference $\left(B_{\text {int }}=170 \mathrm{kHz}\right)$ as in Figure 8, the algorithm is found to reach the steady state after $2.5 \times 10^{6}$ iterations (about $0.153 \mathrm{~s})$. For the narrower interference band $\left(B_{\text {int }}=25\right.$ $\mathrm{kHz}$ ) in Figure 9, the time requirement is shorter with $10^{6}$ iterations (about $0.06 \mathrm{~s}$ ). The bandwidth of some unintentional interference sources for L1 band is under $100 \mathrm{kHz}$. For example, the Aircraft Communication Addressing and Reporting System (ACARS) with $25 \mathrm{kHz}$,

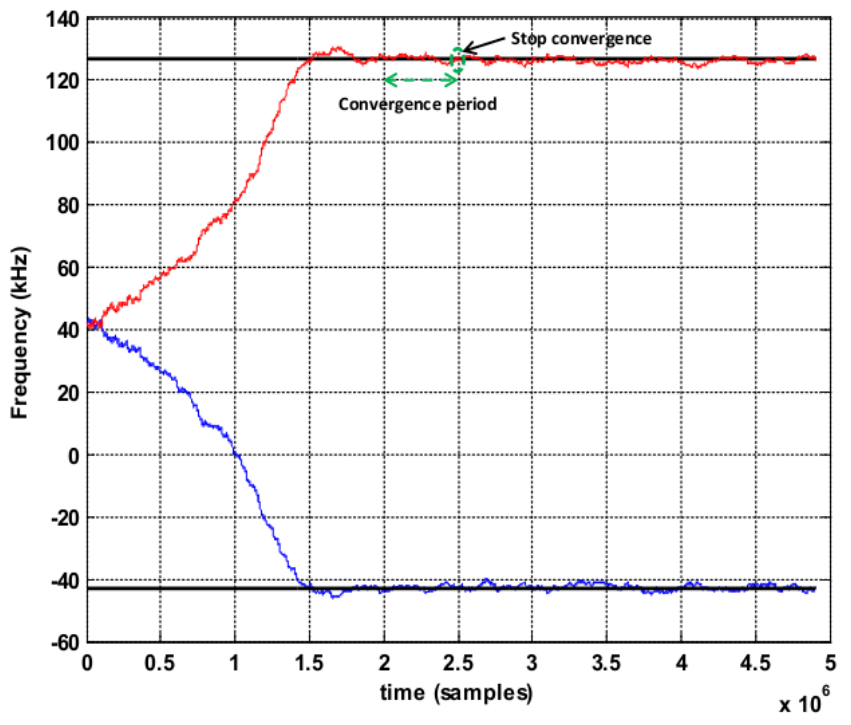

Figure 8. Simulation results: estimation of the interference bandwidth $\left(B_{\text {int }}=170 \mathrm{kHz}\right)$.

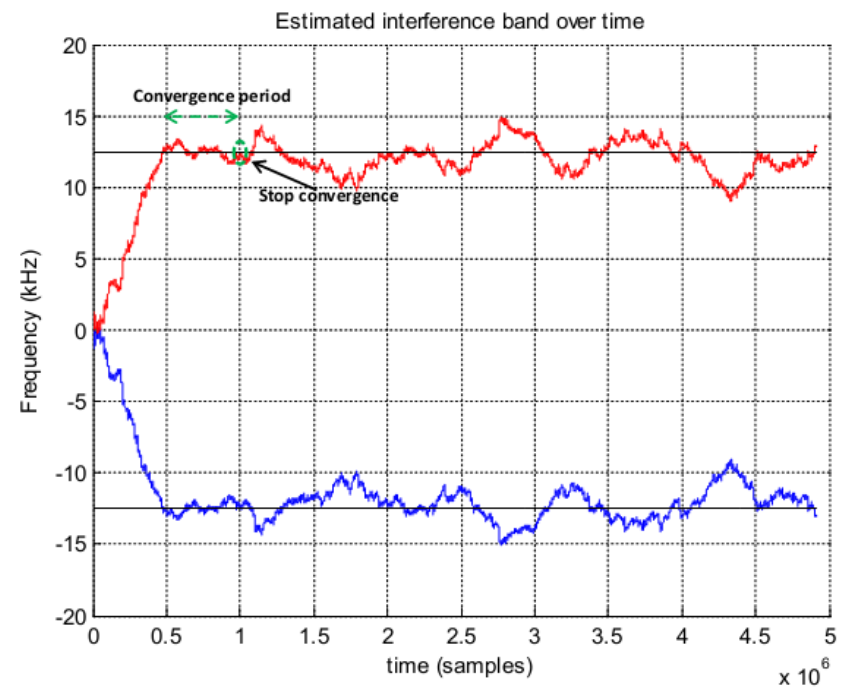

Figure 9. Simulation results: estimation of the interference bandwidth $\left(B_{\text {int }}=25 \mathrm{kHz}\right)$.

and the SATCOM Communications with $20 \mathrm{kHz}$ [16]. Thus, the rate of convergence as the results is acceptable.

Furthermore, the performance of the algorithm has also been tested in case of variable the interference bandwidth. Figure 10 shows the results when the algorithm is applied to a data set of $1 \mathrm{~s}$ containing an interference source, whose characteristics vary over time (i.e., the interference bandwidth is $10 \mathrm{kHz}$ over the first $500 \mathrm{~ms}$, and $18 \mathrm{kHz}$ over the last $500 \mathrm{~ms}$ of simulation).

Although the proposed bandwidth estimation block is able to estimate interference band, it has a limit related to the selection of prefixed bandwidth of ANF 1 and ANF 2. It can be seen in Figure 11, which shows the results of the interference bandwidth estimation for different choices of NF bandwidth. The red line indicates the real interference bandwidth, i.e., $B_{\text {int }}=170 \mathrm{kHz}$. It is worth noticing how the algorithm is able to finely 


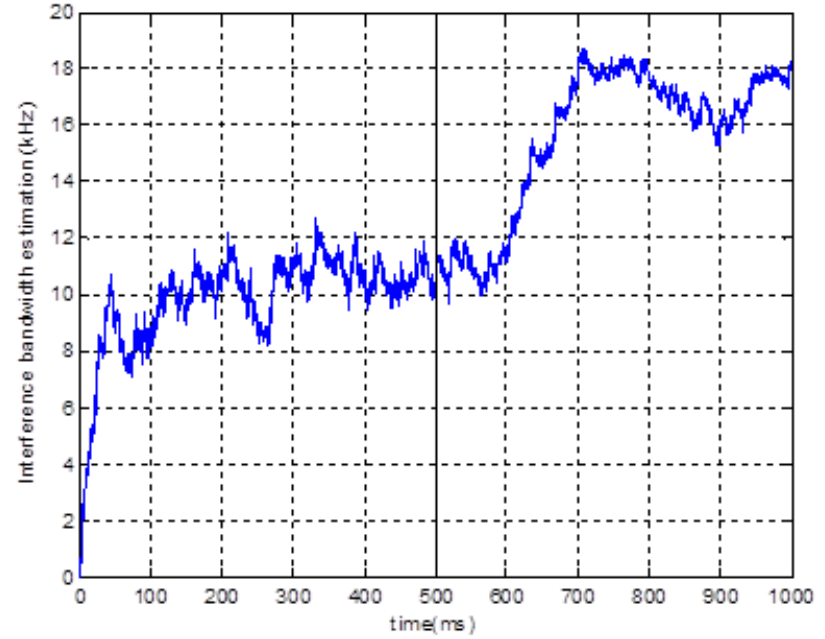

Figure 10. Estimated interference bandwidth over time, in case of variable band.

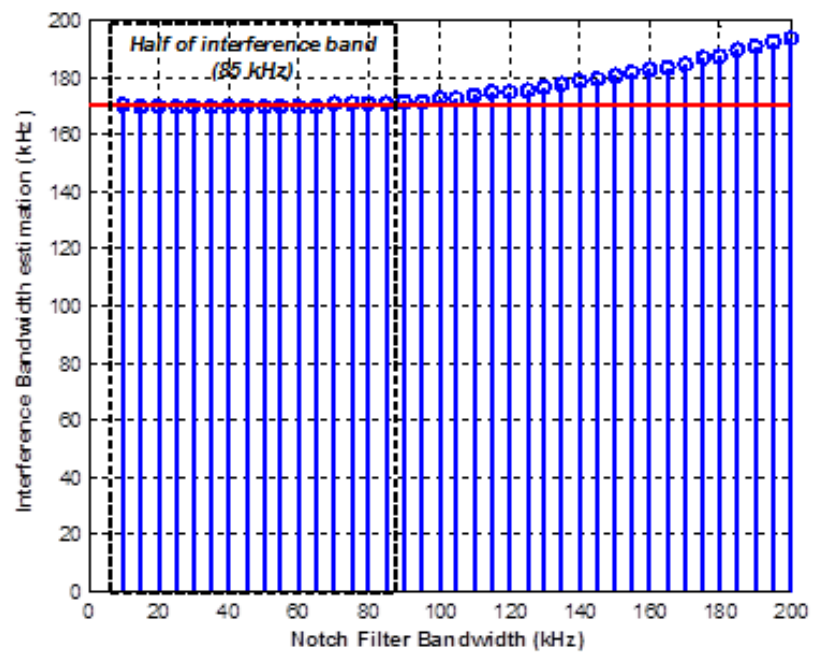

Figure 11. Interference bandwidth estimation for different NFs bandwidth.

estimate the bandwidth, when the width of the NFs bands is less then half of $B_{\text {int }}$.

\subsection{Notch Filter Parameters Adaptation}

In [17], the authors recommended that $k_{\alpha}$ should be set at 0.9 to provide good performance, whereas, in [13], $k_{\alpha}=0.99$ is recommended. In this section, a comparison the quality of the signal after filtering by NFs with three different $k_{\alpha}$ is given, where two are recommended in [17] and [13], and the other is calculated by the proposed method in Section 3. The sequence number of codes and Doppler shift frequency of the satellites presented in the input signal are chosen randomly. The interference frequency matches the intermediate frequency: $f_{\text {int }}=f_{\mathrm{IF}}$. The used method to estimate $C / N_{0}$ is Signal to Noise Variance [18].

It can be seen from the Table I that a too narrow notch does not remove enough the interference power; then it might lead to the signal loss of track. Whereas, a too wide notch leads to the loss of the useful signal, then reduces the value of $C / N_{0}$. In the presence of the proposed interference mitigation module, the quality of the signal is improved more than $1 \mathrm{~dB}$ compared to the cases of $k_{\alpha}=0.9$ and $k_{\alpha}=0.99$. This is because the removed power of the interference is balanced with the unwanted loss of the useful signal in presence of the proposed module. Therefore, a module to tune this factor, as proposed in Section 3, is necessary.

\section{Conclusion}

Narrow-band interferers can be classified among the most dangerous threats for GNSS receivers. For this kind of disturbing signals notch filtering has proved to be an efficient mitigation technique, because it can be considered a good compromise between efficiency and complexity.

The use of NF in interference mitigation requires the knowledge of interference characteristics. Much research in the past only focused on determining the interference frequency. In the current work, we proposed a technique that aims at estimating and tracking the bandwidth of the filter. Then, an expression of $C / N_{0}$ was given to evaluate the quality of the filtered signal. It is also used to optimize the configuration of NF.

Simulation results showed that the proposed methods can estimate the bandwidth of interference and choose an appropriate pole factor for NF. Though some open issues still have to be solved, the promising outcomes give the motivation for further investigations. Future work will mainly focus on the tuning of the bandwidth of the two notch filters used for the interference characterization.

\section{Appendix A}

\section{Proof of Equation (8)}

The received signal can be expressed by

$$
s(t)=g(t)+w(t),
$$

where $g(t)$ is GNSS signal; $w(t)$ is noise, which includes white noise and interference.

Assume that the baseband signal is processed by a correlator, with the integration time $\mathrm{T}$ and local code $m(t)$, the correlator output of each component is:

$$
\begin{aligned}
R_{\mathcal{S}}(0) & =\int_{-\frac{T}{2}}^{\frac{T}{2}} g(t) m(t) d t, \\
R_{w}(\tau) & =\int_{-\frac{T}{2}}^{\frac{T}{2}} w(t) m(t-\tau) d t .
\end{aligned}
$$

According to Plancherel theorem [19], correlation of the useful signal can be written as

$$
\begin{aligned}
R_{S}(0) & =\int_{-\frac{T}{2}}^{\frac{T}{2}} g(t) m(t) d t \\
& =\int_{-\frac{F s}{2}}^{\frac{F s}{2}} X(f) \overline{M(f)} d f,
\end{aligned}
$$


Table I

$C / N_{0}$ of GPS Signals After Filtering by NF. The Interference Bandwidth $B_{\text {int }}=25 \mathrm{kHz}$

\begin{tabular}{|c|c|c|c|c|c|c|}
\hline \multirow{2}{*}{$P_{i}$} & \multirow{2}{*}{ PRN } & \multirow{2}{*}{ Doppler shift } & \multirow{2}{*}{$k_{\alpha}=0.9$} & \multirow{2}{*}{$k_{\alpha}=0.99$} & \multicolumn{2}{|r|}{ Proposed Method } \\
\hline & & & & & $\mathrm{C} / \mathrm{NO}$ & $\hat{B}_{\text {int }}, k_{\alpha}^{\text {optimize }}$ \\
\hline \multirow{4}{*}{$-126.5 \mathrm{~dB}$} & 1 & 2500 & 38.90 & 34.79 & 38.85 & \multirow{4}{*}{$\hat{B}_{\text {int }}=23.6 \mathrm{kHz}, k_{\alpha}=0.965$} \\
\hline & 5 & 1600 & 39.26 & 35.23 & 39.41 & \\
\hline & 7 & 5000 & 38.68 & Loss of track & 38.59 & \\
\hline & 12 & 4200 & 38.93 & 35.23 & 38.95 & \\
\hline \multirow{4}{*}{$-135.5 \mathrm{~dB}$} & 1 & 2500 & 40.16 & 40.82 & 41.66 & \multirow{4}{*}{$\hat{B}_{\text {int }}=25.1 \mathrm{kHz}, k_{\alpha}=0.98$} \\
\hline & 5 & 1600 & 39.85 & 41.29 & 41.91 & \\
\hline & 7 & 5000 & 39.85 & 40.97 & 41.61 & \\
\hline & 12 & 4200 & 40.19 & 41.25 & 42.07 & \\
\hline
\end{tabular}

where $F_{s}$ is the sampling frequency, $X(f)$ and $M(f)$ are Fourier transforms of $g(t)$ and $m(t)$, respectively. $\overline{M(f)}$ denotes the complex conjugation.

If the received signal goes through a filter with transfer function $H(f),(14)$ can be rewritten as

$$
R_{S}(0)=\int_{-\frac{F s}{2}}^{\frac{F_{S}}{2}} H(f) X(f) \overline{M(f)} d f,
$$

where $C_{s}$ is the post-correlation power of GNSS signal, $S(f)$ denotes normalized power spectrum of the GNSS signal. Since $g(t)$ is modulated by the pseudo-range code, the power of useful signal pre-correlation is

$$
\begin{aligned}
P_{s} & =\left|R_{s}(0)\right|^{2} \\
& =\left.\left.2 C_{s}\left|\int_{-\frac{F s}{2}}^{\frac{F s}{2}} H(f)\right| M(f)\right|^{2} d f\right|^{2} \\
& =2 C_{s}\left|\int_{-\frac{F s}{2}}^{\frac{F s}{2}} H(f) S(f) d f\right|^{2} l .
\end{aligned}
$$

For noises, pre-correlation power of noises can be expressed

$$
P_{n}=\frac{1}{T} \int_{-T / 2}^{T / 2}(R(\tau))^{2} d \tau
$$

According to Parseval's theorem [19] and crosscorrelation theorem:

$$
\begin{aligned}
P_{w} & =\frac{1}{T} \int_{-T / 2}^{T / 2}(R(\tau))^{2} d \tau \\
& =\frac{1}{T} \int_{-\frac{F s}{2}}^{\frac{F s}{2}}|\mathcal{F}(R(\tau))|^{2} d f \\
& =\frac{1}{T} \int_{-\frac{F s}{2}}^{\frac{F s}{2}}|H(f) W(f) M(f)|^{2} d f .
\end{aligned}
$$

It is possible to rewrite $|W(f)|^{2}$ as $N_{0}+C_{i} S_{i}(f)$, where $N_{0}$ is the white noise power density, $C_{i}$ is the interference power and $S_{i}(f)$ is the normalized power spectrum of interference.

From (16) and (18), pre-correlation signal to noise ratio is:

$$
\frac{S N R=}{\frac{\frac{2 T C_{s}}{N_{0}}\left|\int_{-\frac{F s}{2}}^{\frac{F s}{2}} H(f) S(f) d f\right|^{2}}{\int_{-\frac{F s}{2}}^{\frac{F s}{2}}|H(f)|^{2} S(f) d f+\frac{C_{i}}{N_{0}} \int_{-\frac{F s}{2}}^{\frac{F s}{2}}|H(f)|^{2} S(f) S_{i}(f) d f} .}
$$

According to [18], the $C / N_{0}$ ratio can be calculated as:

$$
\frac{C}{N_{0}}=\frac{S N R}{T},
$$

where $\mathrm{T}$ is the correlator's integration time.

Thus, applying (19) and (20), we have

$$
\begin{aligned}
& \frac{C}{N_{0}}= \\
& \frac{\frac{2 C_{S}}{N_{0}}\left|\int_{-\frac{F s}{2}}^{\frac{F s}{2}} H(f) S(f) d f\right|^{2}}{\int_{-\frac{F s}{2}}^{\frac{F s}{2}}|H(f)|^{2} S(f) d f+\frac{C_{i}}{N_{0}} \int_{-\frac{F s}{2}}^{\frac{F s}{2}}|H(f)|^{2} S(f) S_{i}(f) d f} .
\end{aligned}
$$

Assume that the interference spectrum is concentrated in the range $\left[f_{\text {int }}-\frac{B_{\text {int }}}{2} ; f_{\text {int }}+\frac{B_{\text {int }}}{2}\right]$, where $B_{\text {int }}$ is the bandwidth of interference and $f_{\text {int }}$ is the interference frequency. Since the signal spectrum is symmetric, (21) can be rewritten as:

$$
\frac{C}{N_{0}}=\frac{\frac{C_{s}}{N_{0}}\left|\int_{0}^{\frac{F_{s}}{2}} H(f) S(f) d f\right|^{2}}{\int_{0}^{\frac{F_{s}}{2}}|H(f)|^{2} S(f) d f+\frac{C_{i}}{N_{0}} \int_{f_{\text {int }}-\frac{B_{\text {int }}}{2}}^{f_{\text {int }}+\frac{B_{\text {int }}}{2}}|H(f)|^{2} S(f) d f} .
$$

\section{ACKNOWLEDGMENT}

This work has been partially funded by the project B2013.01.47. 


\section{REFERENCES}

[1] P. Misra and P. Enge, Global Positioning System: Signals, Measurements and Performance Second Edition. Lincoln, MA: Ganga-Jamuna Press, 2006.

[2] J. V. Carroll, "Vulnerability assessment of the US transportation infrastructure that relies on the global positioning system," The Journal of Navigation, vol. 56, pp. 185-193, May 2003.

[3] E. Backer, D. Van Willigen, and R. Rawlings, “Technical and operational assessment of the suitability of GPS to meet the BRNAV requirements," Delft University of Technology, Telecommunications and Traffic Control Systems Group, 1997.

[4] B. Motella, M. Pini, and F. Dovis, "Investigation on the effect of strong out-of-band signals on global navigation satellite systems receivers," GPS Solutions, vol. 12, no. 2, pp. 77-86, 2008.

[5] F. Bastide, D. Akos, C. Macabiau, and B. Roturier, "Automatic gain control (AGC) as an interference assessment tool," in 16th International Technical Meeting of the Satellite Division of The Institute of Navigation (ION GPS/GNSS 2003), 2003, pp. 2042-2053.

[6] A. T. Balaei, B. Motella, and A. G. Dempster, “GPS interference detected in Sydney-Australia," in IGNSS Symposium, 2007.

[7] E. Kaplan and C. Hegarty, Understanding GPS: principles and applications. Artech house, 2005.

[8] O. Isoz, D. Akos, T. Lindgren, C.-C. Sun, and S.-S. Jan, "Assessment of GPS 11/galileo e1 interference monitoring system for the airport environment," in Proceedings of the 23rd international technical meeting of the Satellite Division of the Institute of Navigation (ION GNSS 2011). Manassas, Va: Inst. of Navigation, Sept 2011.

[9] L. Marti and F. van Graas, "Interference detection by means of the software defined radio," in Proceedings of the 17th International Technical Meeting of the Satellite Division of The Institute of Navigation (ION GNSS 2004), 2004, pp. 99-109.

[10] G. X. Gao, D. S. De Lorenzo, T. Walter, and P. Enge, “Acquisition and Tracking of GIOVE-A Broadcast L1/E5/E6 Signals and Analysis of DME/TACAN Interference on Receiver Design," Proc. ENC 2007, 2007.

[11] D. Borio, L. Camoriano, and L. L. Presti, "Two-pole and multi-pole notch filters: a computationally effective solution for GNSS interference detection and mitigation," IEEE Systems Journal, vol. 2, no. 1, pp. 38-47, 2008.

[12] G. Giordanengo, "Impact of notch filtering on tracking loops for GNSS applications," Master's thesis, Politecnico di Torino, Italy, 2009.

[13] R. J. Landry, V. Calmettes, and M. Bousquet, "Impact of interference on a generic gps receiver and assessment of mitigation techniques," in IEEE 5th International Symposium on Spread Spectrum Techniques and Applications, vol. 1. IEEE, 1998, pp. 87-91.

[14] E. Falletti, D. Margaria, M. Nicola, G. Povero, and M. T. Gamba, "N-fuels and soprano: Educational tools for simulation, analysis and processing of satellite navigation signals," in IEEE Frontiers in Education Conference. IEEE, 2013, pp. 303-308.

[15] L. Tan, J. Jiang, and L. Wang, Adaptive Harmonic IIR Notch Filters for Frequency Estimation and Tracking. INTECH Open Access Publisher, 2011.

[16] R. Landry and A. Renard, "Analysis of potential interference sources and assessment of present solutions for GPS/GNSS receivers," 4th Saint-Petersburg on INS, pp. $1-13,1997$.

[17] J. Raasakka and M. Orejas, "Analysis of notch filtering methods for narrowband interference mitigation," in Position, Location and Navigation Symposium-PLANS 2014, 2014 IEEE/ION. IEEE, 2014, pp. 1282-1292.

[18] M. Pini, E. Falletti, and M. Fantino, "Performance evaluation of $C / N_{0}$ estimators using a real time GNSS software receiver," in IEEE 10th International Symposium on Spread Spectrum Techniques and Applications (ISSSTA'08). IEEE, 2008, pp. 28-31.

[19] W. M. Siebert, Circuits, signals, and systems. MIT press, 1986, vol. 2.

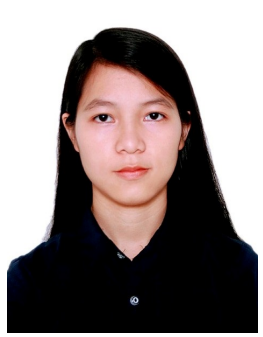

Tu Thi-Thanh Nguyen was born in 1989. She received the Engineer Degree (2012) and the Master Degree (2015) in Information Technology from the School of Information and Communication Technology, Hanoi University of Science and Technology (HUST), Vietnam. Since July, 2012, she has been working in the International Collaboration Center for Research and Development of Satellite Navigation Technology in South-East Asia (NAVIS Centre), Hanoi, Vietnam. Her research activity is focused on GNSS signal processing.

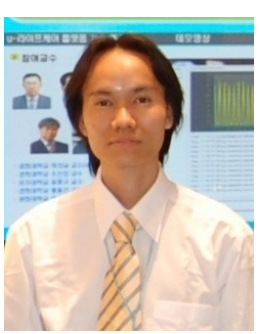

Vinh La The graduated in 2004 and received master degree in 2008 from Hanoi University of Science and Technology (HUST). He completed his PhD at Kyung Hee University, South Korea in June 2012. In recent years and in collaboration with his NAVIS colleagues, he has invested in the research of precise positioning algorithms.

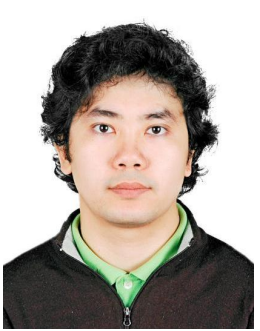

Tung Hai Ta received his Engineer's degree (2003) and M.Sc. degree (2005) in Information Technology from HUST followed by Master degree in Navigation and Related Application (2006) and PhD degree in Information and Communication Technologies (2010) from Politecnico di Torino (PoliTo), Italy. In 2009, he was with Satellite Navigation \& Positioning Laboratory (SNAP Lab), University of New South Wales. From Fevruary 2010 to February 2011, he worked as a PostDoc fellow at Electronics Department, PoliTo. Since March 2011, he has worked at School of Information and Communication Technology, HUST as well as NAVIS Centre. Since December 2011, he has been the Director of NAVIS Centre.

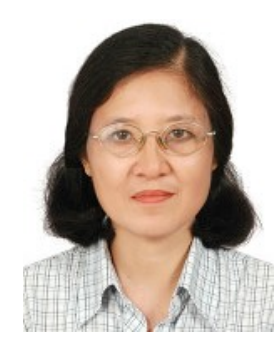

Hoang-Lan Thi Nguyen is working at Department of Data communications and Computer Networks, School of Information and Communication Technology (SoICT), Hanoi University of Science and Technology (HUST), Vietnam. 


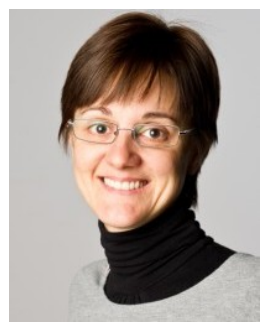

Beatrice Motella received the Master Degree in Communications Engineering in January 2003 at Politecnico di Torino. In December 2007, she completed the Ph.D. program in Electronics and Communications Engineering at the same university, working in the Navigation Signal Analysis and Simulation (NavSAS) group, under the tutoring of Prof. Letizia Lo Presti. During the Ph.D. program she spent almost one year as a visiting researcher at the Satellite Navigation \& Positioning Laboratory (SNAPlab) of the School of Surveying \& Spatial Information Systems, within the University of New South Wales, in Sydney, Australia.

She is currently a researcher at the Istituto Superiore Mario Boella, working in the GNSS Platforms for Safety and Environment unit, within the Navigation Technologies area. 